\title{
Avaliação do TOPMODEL na estimativa do escoamento superficial em microbacia hidrográfica em diferentes usos
}

\author{
Maycon P. Hollanda ${ }^{1}$, Roberto A. Cecílio ${ }^{2}$, Wesley A. Campanharo ${ }^{3}$, Sidney S. Zanetti ${ }^{2}$, \\ Larice N. de Andrade ${ }^{4}$ G Giovanni de O. Garcia ${ }^{5}$ \\ ${ }^{1}$ Faculdade da Região Serrana. Santa Maria de Jetibá, ES. E-mail: mphollanda@hotmail.com \\ ${ }^{2}$ Departamento de Ciências Florestais e da Madeira/Universidade Federal do Espírito Santo. Jerônimo Monteiro, ES. E-mail: roberto.cecilio@ufes.br \\ (Autor correspondente); sszanetti@yahoo.com.br \\ ${ }^{3}$ Programa de Pós-graduação em Ciências Florestais/Universidade Federal do Espírito Santo. Jerônimo Monteiro, ES. E-mail: wesley-ac@hotmail.com \\ ${ }^{4}$ Departamento de Computação/Universidade Federal do Espírito Santo. Alegre, ES. E-mail: larice.andrade@ufes.br \\ ${ }^{5}$ Departamento de Engenharia Rural/Universidade Federal do Espírito Santo. Alegre, ES. E-mail: giovanni@cca.ufes.br
}

\section{Palavras-chave: \\ deflúvio \\ modelagem hidrológica \\ manejo de bacias hidrográficas}

\begin{abstract}
R E S U M O
Avaliou-se a aplicabilidade do modelo hidrológico TOPMODEL em simular os hidrogramas de escoamento superficial gerado por eventos isolados de precipitação em uma microbacia hidrográfica sob manejo conservacionista. Utilizaram-se de características físicas, hidrológicas e edáficas da microbacia associadas a hidrogramas de escoamento superficial relativos a eventos de vazão e precipitação medidos entre 2009 e 2012 . Observou-se que o TOPMODEL mostrou tendência em subestimar a vazão máxima e a lâmina total de escoamento superficial e se constatou, todavia, que o TOPMODEL é promissor na modelagem dos hidrogramas de escoamento superficial de eventos de chuva isolados na microbacia sob manejo conservacionista em estudos, embora tenda a subestimar os valores de vazão.
\end{abstract}

\section{Key words:}

streamflow

hydrologic modelling

watershed management

\section{Evaluation of TOPMODEL for prediction of the runoff of a watershed under different land uses}

\begin{abstract}
A B S T R A C T
The applicability of TOPMODEL hydrologic model to predict the runoff hydrographs originated from isolated rainfall events over a small watershed under conservationist management practices was evaluated. Geographical, hydrological and soil data from the watershed were used associated to streamflow hydrographs and rainfall events measured between 2009 and 2012. TOPMODEL mainly underestimated runoff peak flow and runoff amount. However, it was observed that TOPMODEL is promising for the estimation of runoff hydrograph originated from isolated rainfall events at the studied small watershed under conservationist management practices.
\end{abstract}




\section{INTRODUÇÃo}

As microbacias hidrográficas consistem nas unidades naturais ideais para a gestão dos recursos naturais e implementação de políticas públicas conservacionistas e ambientais, haja vista que é nessas unidades que ocorrem os processos relacionados com o deslocamento das águas na superfície terrestre, a erosão hídrica e as atividades agropecuárias e florestais, com destaque para suas interrelações e impactos sobre os recursos naturais, especialmente sobre o solo e as águas.

As atividades agroflorestais de caráter conservacionista devem considerar a dinâmica das águas nas microbacias e adotar práticas conservacionistas de produção de modo a não comprometer a capacidade produtiva do solo nem a qualidade das águas, com repercussões sobre a produção de alimentos, fibras e sobre o meio ambiente.

A utilização de modelos hidrológicos proporciona custos menores e economia de tempo para analisar os potenciais impactos das mudanças antrópicas no regime hídrico de bacias hidrográficas. Os modelos hidrológicos são ferramentas primordiais para avaliar, simular e prever os danos favorecidos por eventos de precipitação auxiliando no planejamento, no manejo e na tomada de decisões relacionadas aos recursos naturais, especialmente os hídricos, em uma bacia hidrográfica. Neste sentido são ferramentas fundamentais para se planejar o manejo conservacionista de microbacias hidrográficas.

Diversos modelos hidrológicos foram e estão sendo desenvolvidos pela comunidade científica, dentre os quais se destaca o TOPMODEL (Topography Based Hydrological Model) desenvolvido por Beven \& Kirkby (1979). Os principais atrativos na aplicação do TOPMODEL estão relacionados à necessidade de conhecimento de um pequeno número de parâmetros de entrada associado a um razoável embasamento teórico. Desde sua elaboração o modelo TOPMODEL foi aplicado em bacias hidrográficas sob diferentes usos e condições edafoclimáticas e de uso do solo (Mine \& Clarke, 1996; Wu et al., 2007; Huiping \& Shufen, 2010; Lin et al., 2010; Gumindoga et al., 2011; Nourani et al., 2011; Pan \& King, 2012; Qi et al., 2013).

Apesar de haver diversos trabalhos desenvolvidos no Brasil, ainda são poucos, observando-se carência na avaliação da aplicabilidade do TOPMODEL no país, especialmente em microbacias submetidas ao manejo conservacionista. Diante do exposto objetivou-se avaliar a aplicabilidade do modelo hidrológico TOPMODEL em simular os hidrogramas de escoamento superficial gerado por eventos isolados de precipitação em uma microbacia hidrográfica sob manejo conservacionista.

\section{Material e Métodos}

O presente estudo foi desenvolvido na Microbacia Hidrográfica do Córrego Jaqueira (MCJ), pertencente à Bacia Hidrográfica do Rio Itapemirim; possui área de drenagem igual a $0,22 \mathrm{~km}^{2}$, com coordenadas geográficas entre $-20^{\circ} 45^{\prime} 16,2^{\prime \prime}$ e $-20^{\circ} 45^{\prime} 36,72^{\prime \prime}$ de latitude e entre $-41^{\circ} 31^{\prime} 44,76^{\prime \prime}$ e $-41^{\circ} 31^{\prime}$ $25,32^{\prime \prime}$ de longitude; localiza-se próximo à cidade de Alegre ES, em que sua classificação climática é "Cwa" (classificação de Köppen), caracterizada como clima de inverno seco e verão chuvoso, com precipitação total anual média de $1341 \mathrm{~mm}$ e temperatura anual média de $23,1^{\circ} \mathrm{C}$ (Lima et al., 2008).

Historicamente, a ocupação agrícola na MCJ iniciou-se há cerca de 60 anos, com a substituição da mata nativa para implantação de cafeicultura que, posteriormente, cedeu espaço à pastagem extensiva. Há cerca de 15 anos as áreas de topo de morro e próximas ao cursos d'água foram cercadas para dar início ao processo regenerativo da vegetação nativa; atualmente, a área é destinada à preservação; ressalta-se que a pequena parcela de cultivo agrícola é destinada à subsistência cuja prática segue técnicas agroecológicas.

Realizou-se levantamento planialtimétrico da microbacia utilizando-se GPS geodésico estático e se obtendo, posteriormente, o modelo numérico do terreno - MNT (Figura 1A), com pixels com dimensões de 7 x $7 \mathrm{~m}$, uma vez que a versão do modelo TOPMODEL utilizada possui restrição quanto ao número de linhas e colunas do arquivo do MNT. O solo da MCJ é o Argissolo Vermelho-Amarelo ocupado por cinco diferentes sítios (Figura 1B). Os sítios foram classificados da seguinte forma: a) Sítio 1 - ocupando 39\% da MCJ tem, como uso da terra, a pastagem (Brachiaria decumbens); Sítio 2 - ocupado por pastagem (Melinis minutiflora P. Beauv.) com formações arbustivas esparsas; representa 27\% da MCJ; Sítio 3 - uso da terra pelo cultivo do eucalipto (Eucalyptus grandis) sob espaçamento de $3 \times 2 \mathrm{~m}$, com aproximadamente 10 anos de idade, sendo a área igual a 10\% da MCJ; Sítio 4 - ocupado por regeneração florestal em nível secundário ocupando $11 \%$
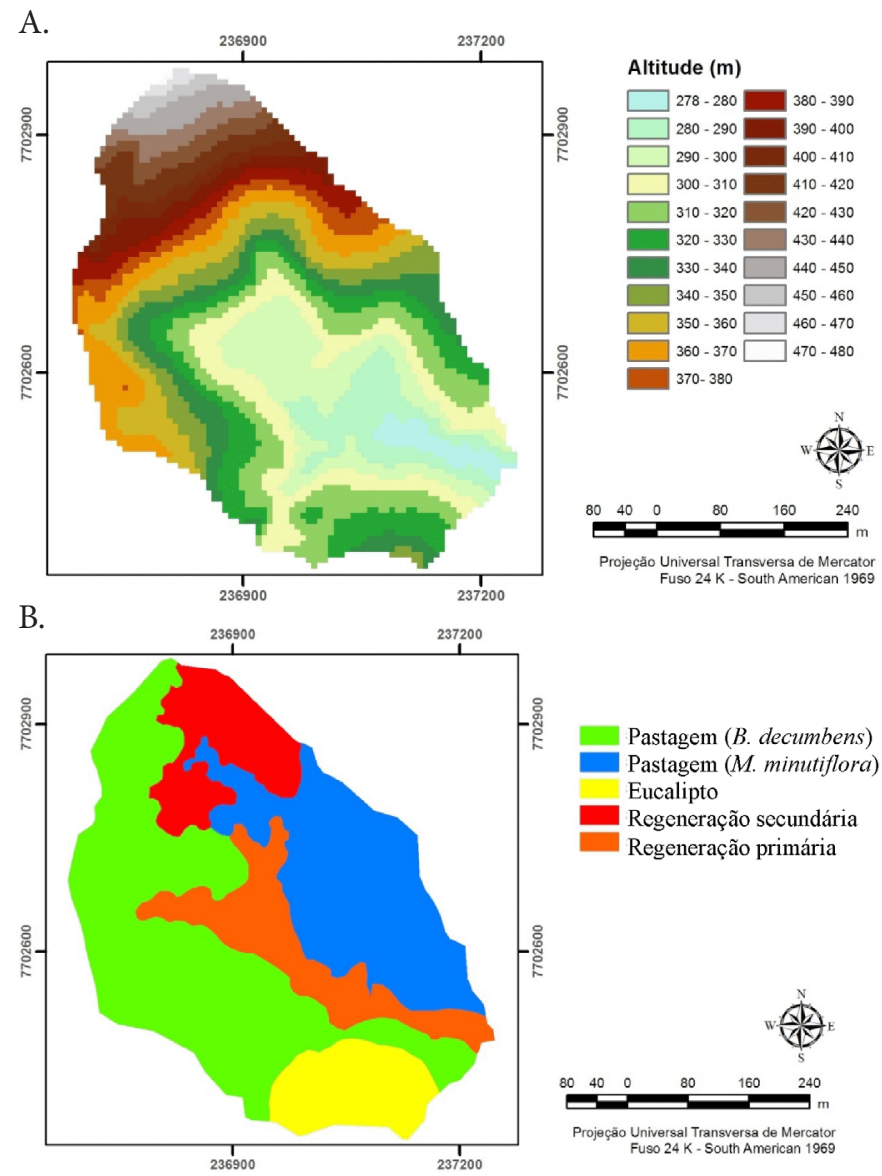

Figura 1. Microbacia Hidrográfica do Córrego Jaqueira: Modelo numérico do terreno (A) e mapa de uso e ocupação do solo (B) 
da área total da MCJ; Sítio 5: ocupado por regeneração vegetal nativa em nível primário formado basicamente por uma única espécie, ocupando $13 \%$ da MCJ.

Para cada sítio foram determinados, por meio das metodologias de EMBRAPA (1997), os atributos físicos dos solos (Tabela 1): granulometria; capacidade de campo, ponto de murcha permanente, densidade do solo, densidade de partículas, macro e microporosidade. A taxa de infiltração estável de água no solo (Tie) foi determinada utilizandose o método de anéis concêntricos (Brandão et al., 2006). Foram feitas cinco repetições de cada determinação em duas profundidades ( 0 a $10 \mathrm{~cm}$ e 10 a $60 \mathrm{~cm}$ ), sendo apresentados, na Tabela 1, os valores médios de cada sítio, pois o TOPMODEL se utiliza apenas de um deles.

O monitoramento da precipitação e vazão na foz da microbacia foi realizado entre março de 2009 e dezembro de 2012, em intervalos de $3 \mathrm{~min}$, por meio de estação meteorológica automática e vertedor triangular dotado de linígrafo de boia. Obtiveram-se hidrogramas de escoamento do curso d'água durante cada evento de precipitação. A lâmina de escoamento superficial e a vazão máxima foram calculadas a partir da separação entre o hidrograma de escoamento superficial e hidrograma de fluxo subterrâneo utilizando-se o método de Barnes (Zanetti et al., 2009).

Para aplicação do TOPMODEL são requeridas, como dados de entrada: precipitação, evapotranspiração potencial e vazão observada (sendo este utilizado para comparação com a vazão simulada). Como o presente trabalho trata da simulação de eventos de curta duração sendo utilizada a precipitação efetiva como dados de entrada, optou-se por atribuir valor zero à evapotranspiração, como adotado por Castilho (2005).

O modelo utiliza um único parâmetro distribuído espacialmente: o índice topográfico (IT). O IT foi calculado por meio do software DTM 97.07 utilizando-se o MNT como entrada e o algoritmo de direção de fluxo (Quinn et al., 1995) para o cálculo (Eq. 1).

$$
\mathrm{IT}=\ln \frac{\mathrm{a}_{\mathrm{i}}}{\operatorname{tg} \beta_{\mathrm{i}}}
$$

em que:

IT - índice topográfico adimensional

$a_{i} \quad$ - área de drenagem específica por unidade de contorno para o pixel $\mathrm{i}, \mathrm{m}^{2} \mathrm{~m}^{-2}$

$\operatorname{tg} \beta_{\mathrm{i}}$ - declividade média do terreno calculada no ponto $i$, $\mathrm{m} \mathrm{m}^{-1}$
O modelo considera, ainda, cinco parâmetros de entrada, apresentados na Tabela 2. A capacidade máxima de água disponível na zona radicular $\left(\mathrm{SR}_{\text {máx }}\right)$, que foi calculada a partir dos valores de capacidade de campo, ponto de murcha permanente e profundidade efetiva do sistema radicular das culturas. $\mathrm{O}$ valor único de $\mathrm{SR}_{\text {máx }}$ foi obtido através da média ponderada para os diferentes tipos de cobertura vegetal da bacia. O déficit inicial de umidade na zona radicular $\left(\mathrm{SR}_{\mathrm{init}}\right)$ foi tomado como $40 \%$ de $\mathrm{SR}_{\text {máx }}$ (Ferreira, 2004). Este procedimento foi adotado levando-se em conta que referido parâmetro depende tão somente dos eventos anteriores; considerou-se, então, inicialmente, que as condições de umidade dos solos fossem intermediárias.

Tabela 2. Parâmetros de entrada do modelo hidrológico TOPMODEL

\begin{tabular}{clc}
\hline Parâmetro & \multicolumn{1}{c}{ Descrição } & Unidade \\
$\mathrm{m}_{0}$ & Armazenamento exponencial & $\mathrm{m}$ \\
$\mathrm{T}_{0}$ & Transmissividade efetiva do solo saturado & $\mathrm{m}^{2} \mathrm{~h}^{-1}$ \\
$\mathrm{SR}_{\operatorname{máx}}$ & Capacidade de água disponível na zona radicular & $\mathrm{m}$ \\
$\mathrm{SR}_{\text {init }}$ & Déficit inicial de umidade na zona radicular & $\mathrm{m}$ \\
ChVel & Velocidade de propagação em canal & $\mathrm{m} \mathrm{h}^{-1}$ \\
\hline
\end{tabular}

A velocidade de propagação em canal (ChVel) foi calculada pelas Eq. 2 e 3

$$
\begin{gathered}
\text { ChVel }=\frac{\mathrm{d}}{\mathrm{t}_{\mathrm{c}}} \\
\mathrm{t}_{\mathrm{c}}=\frac{57}{60}\left(\frac{\mathrm{L}^{2}}{\mathrm{H}}\right)^{0,385}
\end{gathered}
$$

em que:

d - comprimento do canal principal da bacia, $m$

$t_{c}$ - tempo de concentração calculado pela equação de Kirpich (Eq. 3), h

L - comprimento do talvegue, $\mathrm{km}$

$\mathrm{H}$ - diferença de nível entre o ponto mais remoto e a seção de deságue, $m$

Por não se ter medidas de campo, $\mathrm{m}_{0} \mathrm{e} \mathrm{T}_{0}$ foram estimados com base nas características hidrológicas e edáficas da MCJ e com suporte em valores utilizados por diversos autores (Franchini et al., 1996; Schuler et al., 2000; Beven \& Freer, 2001; Ferreira, 2004; Santos \& Kobiyama, 2008) e posteriormente

\begin{tabular}{|c|c|c|c|c|c|}
\hline \multirow{2}{*}{ Atributo } & \multicolumn{5}{|c|}{ Sítio } \\
\hline & 1 & 2 & 3 & 4 & 5 \\
\hline Teor de areia $\left(\mathrm{g} \mathrm{kg}^{-1}\right)$ & 490,75 & 482,16 & 467,48 & 465,49 & 618,34 \\
\hline Teor de silte $\left(\mathrm{g} \mathrm{kg}^{-1}\right)$ & 96,48 & 72,25 & 102,11 & 135,27 & 91,78 \\
\hline Teor de argila $\left(\mathrm{g} \mathrm{kg}^{-1}\right)$ & 412,77 & 445,60 & 430,41 & 399,24 & 289,88 \\
\hline Classificação textural & Argilosa & Argilosa & Argilosa & Argilosa & Média \\
\hline Capacidade de campo $\left(\mathrm{m}^{3} \mathrm{~m}^{-3}\right)$ & 0,2433 & 0,2534 & 0,2588 & 0,2611 & 0,2136 \\
\hline Ponto de murcha permanente $\left(\mathrm{m}^{3} \mathrm{~m}^{-3}\right)$ & 0,1928 & 0,2057 & 0,2034 & 0,2040 & 0,1520 \\
\hline Densidade do solo $\left(\mathrm{kg} \mathrm{dm}^{-3}\right)$ & 1,39 & 1,36 & 1,35 & 1,35 & 1,48 \\
\hline Densidade de partículas $\left(\mathrm{kg} \mathrm{dm}^{-3}\right)$ & 2,60 & 3,05 & 2,68 & 2,91 & 2,47 \\
\hline Microporosidade $\left(\mathrm{m}^{3} \mathrm{~m}^{-3}\right)$ & 0,31 & 0,32 & 0,33 & 0,34 & 0,27 \\
\hline Macroporosidade $\left(\mathrm{m}^{3} \mathrm{~m}^{-3}\right)$ & 0,15 & 0,24 & 0,18 & 0,19 & 0,14 \\
\hline Taxa de infiltração estável $\left(\mathrm{mm} \mathrm{h}^{-1}\right)$ & 50,1 & 163,4 & 184,1 & 119,0 & 439,1 \\
\hline
\end{tabular}
calibrados.

Tabela 1. Atributos do solo nos sítios da Microbacia Hidrográfica do Córrego Jaqueira 
Para a avaliação do TOPMODEL foram utilizados eventos de chuva os quais geraram escoamento superficial observado (Tabela 3) ao longo do período de coleta de dados, os quais foram separados em amostras para calibração e validação. Para a calibração foram selecionados, aleatoriamente, os eventos P2, P4, P10, P11 e P13, os quais representam aproximadamente $31 \%$ do total de eventos disponíveis.

Tabela 3. Eventos de precipitação usados na avaliação do TOPMODEL na MCJ

\begin{tabular}{|c|c|c|c|c|}
\hline \multirow{2}{*}{ Evento } & \multirow{2}{*}{$\begin{array}{c}\text { Duração } \\
\text { (min) }\end{array}$} & \multirow{2}{*}{$\begin{array}{c}\text { PT } \\
(\mathrm{mm})\end{array}$} & $I p_{\text {máx. }}$ & $I p_{\text {méd }}$ \\
\hline & & & \multicolumn{2}{|c|}{$\left(\mathrm{mm} \mathrm{h}^{-1}\right)$} \\
\hline P1 & 99 & 21,3 & 106,6 & 12,7 \\
\hline P2 & 141 & 13,7 & 30,4 & 5,8 \\
\hline P3 & 123 & 19,3 & 40,6 & 9,2 \\
\hline P4 & 159 & 19,3 & 45,7 & 7,2 \\
\hline P5 & 72 & 22,8 & 66,0 & 18,8 \\
\hline P6 & 33 & 19,0 & 81,2 & 34,6 \\
\hline P7 & 165 & 28,4 & 45,7 & 10,3 \\
\hline P8 & 48 & 24,4 & 111,7 & 30,1 \\
\hline P9 & 258 & 35,8 & 91,4 & 8,3 \\
\hline P10 & 90 & 28,9 & 96,5 & 19,1 \\
\hline P11 & 54 & 20,6 & 116,8 & 22,0 \\
\hline P12 & 195 & 38,8 & 66,0 & 11,9 \\
\hline P13 & 120 & 25,1 & 50,8 & 12,5 \\
\hline P14 & 141 & 34,8 & 45,7 & 14,8 \\
\hline P15 & 21 & 11,1 & 66,0 & 31,2 \\
\hline P16 & 129 & 32,8 & 86,3 & 17,7 \\
\hline
\end{tabular}

PT - Precipitação total; $I p_{\text {máx }}$ - Intensidade de precipitação máxima; $I p_{\text {méd. }}$ - Intensidade de precipitação média

Foram calibrados os parâmetros $\mathrm{m}_{0} \mathrm{e} \mathrm{T}_{0}$, que são aqueles para os quais o TOPMODEL apresenta maior sensibilidade, segundo Varella \& Campana (2000), Gumindoga et al. (2011) e Bhaskar et al. (2005). A determinação dos melhores valores desses parâmetros se constituiu através da geração de mil simulações aleatórias pelo método de Monte Carlo dos intervalos sugeridos na literatura (Franchini et al., 1996; Schuler et al., 2000; Beven \& Freer, 2001; Ferreira, 2004; Santos \& Kobiyama, 2008) de $\mathrm{m}_{0}$ (entre 0,00315 e 1,4999 $\mathrm{m}$ ) e de $\mathrm{T}_{0}$ (entre 0,0009 e $12981 \mathrm{~m}^{2} \mathrm{~h}^{-1}$ ). Após a obtenção do conjunto de $\mathrm{m}_{0}$ e $\mathrm{T}_{0}$ que apresentaram a melhor concordância de cada evento na calibração, foi obtido um valor médio de cada parâmetro determinando, assim, os valores calibrados.

O processo de validação consistiu na utilização dos parâmetros calibrados para a simulação dos 14 eventos restantes, sem realizar nenhum tipo de ajuste adicional.

Para a análise da qualidade de uma simulação foram avaliados, para cada evento, os hidrogramas de escoamento superficial e suas respectivas vazões máximas $\left(\mathrm{Q}_{\text {maxx }}\right)$ e lâminas de escoamento superficial (LES). Conforme Qi et al. (2013) e Nourani et al. (2011), o desempenho de estimativa de $Q_{\text {máx }}$ e LES foi calculado em termos dos erros percentuais. A análise do desempenho dos hidrogramas foi realizada pelo coeficiente de eficiência - NS (Nash \& Sutcliffe, 1970).

\section{Resultados e Discussão}

A espacialização do índice topográfico é apresentada na Figura 2. Os valores de IT calculados variaram entre 1,59 e 27,22, com média de 10,11. Valores semelhantes foram encontrados por Silva \& Kobiyama (2007) em uma bacia com $104 \mathrm{~km}^{2}$, na qual o IT variou entre 2,81 e 28,13, com média de

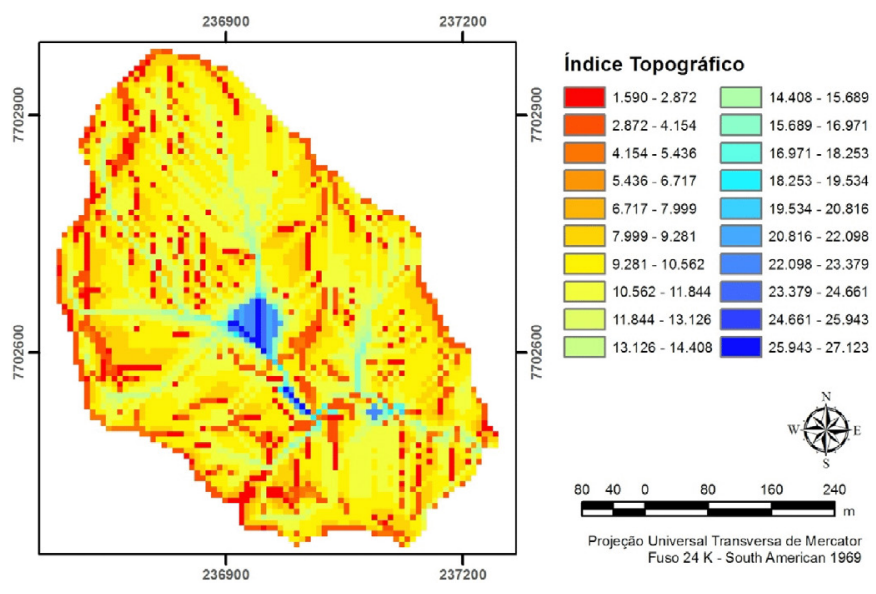

Figura 2. Distribuição espacial do índice topográfico na Microbacia do Córrego Jaqueira

8,00 . Este fato indica que as condições de relevo das bacias são semelhantes mesmo apresentando discrepância em relação ao tamanho da área de drenagem.

Os valores mais altos de IT estiveram associados aos pixels relacionados à rede de drenagem e/ou às depressões do terreno enquanto que os menores valores foram encontrados em regiões com declive mais acentuado visto que o IT é dependente da declividade e da área de contribuição. Os pixels da bacia apresentam altos IT quando o relevo plano está combinado com uma grande área de contribuição. Em contrapartida, elevadas declividades e pequenas áreas de contribuição irão condicionar a baixos valores de IT. Quanto maior o valor do IT maior também será a propensão da bacia em atingir a saturação e gerar escoamento superficial (Siefert, 2012; Kulasova et al., 2014).

Alguns estudiosos avaliaram o comportamento do IT para diferentes resoluções do MNT (Quinn et al., 1995; Varella \& Campana, 2000; Lin et al., 2010; Pan \& King, 2012), mostrando que este apresenta comportamento diferenciado de acordo com a resolução espacial. Na medida em que o tamanho da célula aumenta, os valores do IT tendem a ser mais altos, podendo não representar pequenos canais. Portanto, deve ocorrer um ajuste entre a área superficial, o tamanho de célula e a precisão da informação para melhor representar a bacia na simulação. Ao utilizar um MNT com resolução de 7 x $7 \mathrm{~m}$, observou-se que os valores de IT estão coerentes com as características da microbacia de vez que áreas com elevadas declividades e/ou pequena área de contribuição, apresentam baixo IT.

Para cada evento foi ajustado o melhor conjunto de valores dos parâmetros para estimar as vazões (Tabela 4), procurando um ajuste melhor das hidrógrafas entre os valores simulados e os observados. Os valores médios, apresentados na última linha da Tabela 4, consistem nos valores tomados como calibrados e usados posteriormente na fase de validação do TOPMODEL para a MCJ.

Analisando a Tabela 4, os resultados calculados de $\mathrm{m}_{0}$ para todos os eventos apresentaram valor mínimo de 0,2995 e valor máximo de 0,8390 . Esses valores estão dentro da faixa de valores utilizada por Buytaert et al. (2003), entre 0,0 e 1,0 $\mathrm{m}$ e acima dos valores máximos calculados por vários autores (Schuler et al., 2000; Varella \& Campana, 2000; Gumindoga et al., 2011; Siefert, 2012), de 0,04, 0,20, 0,18 e 0,17 m, 
Tabela 4. Valores dos parâmetros* de entrada do modelo TOPMODEL pertinentes aos eventos de calibração para a Microbacia do Córrego Jaqueira

\begin{tabular}{ccccccc}
\hline Evento & $\mathbf{m}_{\mathbf{0}}$ & $\boldsymbol{I n}\left(\mathbf{T}_{\mathbf{0}}\right)$ & $\mathbf{S R}_{\operatorname{máx}}$ & $\mathbf{S R}_{\text {init }}$ & ChVel & NS \\
P2 & 0,2995 & $-0,8249$ & 0,0545 & 0,0218 & 7212 & 0,1224 \\
P4 & 0,3538 & 3,9727 & 0,0545 & 0,0218 & 7212 & 0,1005 \\
P10 & 0,4193 & $-5,5747$ & 0,0545 & 0,0218 & 7212 & 0,5949 \\
P11 & 0,5011 & 0,4204 & 0,0545 & 0,0218 & 7212 & 0,5636 \\
P13 & 0,8390 & 5,8613 & 0,0545 & 0,0218 & 7212 & 0,2252 \\
\hline Média & 0,4826 & 0,7710 & 0,0545 & 0,0218 & 7212 & - \\
\hline
\end{tabular}

* Para descrição dos parâmetros ver Tabela 2

respectivamente. $\mathrm{O}$ parâmetro $\mathrm{m}_{0}$, de acordo com Beven et al. (1984) e Mine \& Clarke (1996), é o mais importante do modelo para o controle da resposta hidrológica pois influencia as áreas de contribuição e, portanto, a parcela da precipitação que irá tornar-se escoamento superficial. Este parâmetro é o controlador da profundidade efetiva do solo sendo que um valor elevado do mesmo indica um perfil mais condutor de água, enquanto valores inferiores estão associados a uma condutividade menor no perfil do solo. Os solos da MCJ possuem perfis mais profundos os quais, associados ao manejo conservacionista adotado, possuem maiores capacidade de infiltração e transmissão de água (conforme mostra a taxa de infiltração estável na Tabela 1), comparativamente aos solos das bacias anteriormente citadas, o que justifica os maiores valores de $\mathrm{m}_{0}$.

Os valores do parâmetro $\ln \left(\mathrm{T}_{0}\right)$ ajustados para a bacia variaram entre $-5,5747$ e $5,8613 \mathrm{~m}^{2} \mathrm{~h}^{-1}$, escala esta que abrange o valor de $0,6974 \mathrm{~m}^{2} \mathrm{~h}^{-1}$ encontrado por Ferreira (2004), em uma bacia de uso agrícola sob predomínio de argissolos e de 0,11 a 3,50 $\mathrm{m}^{2} \mathrm{~h}^{-1}$ encontrados por Silva \& Kobiyama (2007). Os valores estimados para as condições da MCJ apresentaram alta variação quando comparados aos estimados em outras áreas, o que se deve às diferenças entre tipos e usos do solo deste e dos demais trabalhos. Esta alta variação ainda indica que a condutividade hidráulica nesta bacia apresenta uma variabilidade maior (Tabela 1). Schuler et al. (2000) e Wu et al. (2007) observaram que durante o processo de calibração do modelo TOPMODEL o parâmetro $\mathrm{T}_{0}$ era influenciado pela resolução espacial passível, portanto, de perder o significado físico e atingir valores frequentemente superiores aos obtidos em campo.

O método utilizado para obtenção dos parâmetros $\mathrm{m}_{0}$ e $\mathrm{T}_{0}$ foi satisfatório visto que os valores encontrados estão dentro da faixa de variação da literatura (entre 0,0031 e 1,4999 para $\mathrm{m}_{0}$ e entre $-7,013116$ e 9,47124 pra $\mathrm{T}_{0} \mathrm{~m}^{2} \mathrm{~h}^{-1}$ ) e os valores do NS foram positivos; entretanto, deve-se salientar que o ideal é a obtenção dos parâmetros a partir de medidas a campo, a fim de ter melhor representação hidrológica.

Os parâmetros obtidos pelo método de Monte Carlo com os melhores índices de eficiência foram aplicados para a simulação dos eventos selecionados para o processo de calibração no modelo. Os principais resultados obtidos nessas simulações estão apresentados na Tabela 5. Observa-se, em todos os eventos, que tanto os valores de $\mathrm{Q}_{\text {máx }}$ quanto os de LES simulados subestimaram os observados, o que contribuiu para que a mesma tendência fosse observada na validação, conforme apresentado na sequência. Os erros percentuais médios entre as $\mathrm{Q}_{\text {máx }}$ e LES observadas e simuladas, foram de 64 e 53\%, respectivamente. Mencionados resultados corroboram
Tabela 5. Resultados relativos aos eventos de calibração do TOPMODEL na Microbacia do Córrego Jaqueira, pertinentes à vazão máxima, lâmina (valores pontuais e erro percentual) e hidrograma de escoamento superficial (coeficiente de eficiência - NS)

\begin{tabular}{|c|c|c|c|c|c|c|c|}
\hline \multirow[b]{2}{*}{ Evento } & \multicolumn{3}{|c|}{ Vazão máxima } & \multicolumn{3}{|c|}{ Lâmina } & \multirow[b]{2}{*}{$\begin{array}{l}\text {-Hidrograma } \\
\text { NS (adim.) }\end{array}$} \\
\hline & Simulad & bservada & $\begin{array}{l}\text { Erro } \\
(\%)\end{array}$ & $\underline{\text { Simt }}$ & $\frac{\text { Observada }}{\mathrm{Im})}$ & $\begin{array}{l}\text { Erro } \\
(\%)\end{array}$ & \\
\hline P2 & 0,43 & 1,46 & 71 & 0,02 & 0,05 & 60 & 0,12 \\
\hline P4 & 0,43 & 2,75 & 84 & 0,01 & 0,10 & 90 & 0,1 \\
\hline P10 & 2,14 & 5,27 & 59 & 0,12 & 0,19 & 37 & 0,59 \\
\hline P11 & 2,44 & 7,74 & 68 & 0,06 & 0,27 & 78 & 0,56 \\
\hline $\mathrm{P} 13$ & 0,46 & 0,74 & 38 & 0,03 & 0,03 & 0 & 0,23 \\
\hline
\end{tabular}

Castilho (2005) (subestimativa de $\mathrm{Q}_{\text {máx }}$ em 3\% e LES em 1\%) e Ferreira (2004) (subestimativa da $\mathrm{Q}_{\text {máx }}$ em 19\% e da LES em 19\%), embora tenham apresentado magnitude bastante superior.

Na Tabela 6 são apresentados os resultados obtidos nas simulações dos eventos separados para a validação. Nota-se que os dados simulados de $\mathrm{Q}_{\text {máx }}$ e LES pelo modelo TOPMODEL apresentaram, em relação aos dados observados, valores subestimados na maior parte dos eventos com uma variação entre os valores de $\mathrm{Q}_{\text {máx }}$ de -22 a $78 \%$, sendo a média de $39 \%$. Os resultados foram semelhantes aos encontrados por Ferreira (2004) que também verificou tendência do TOPMODEL em subestimar $\mathrm{Q}_{\text {máx }}$ na faixa de -400 a $50 \%$, sendo o valor médio de $31 \%$. Com relação à LES, o modelo apresentou subestimação em 6 dos 11 eventos; percebe-se, porém, que o modelo possui mais exatidão na simulação da LES, o que pode ser explicado pelo fato da LES ser obtida a partir da divisão do volume de escoamento superficial pela área total da bacia enquanto $\mathrm{Q}_{\text {máx }}$ é um valor pontual do hidrograma de mais difícil estimativa.

Com relação à avaliação dos hidrogramas, observa-se que todos os eventos apresentaram valores positivos de NS; apenas três (P7, P12 e P16) foram superiores a 0,70, o que, de acordo com Mine \& Clarke (1996), indica alta eficiência do TOPMODEL para esses eventos, mesmo que mais três eventos (P5, P6 e P8) tenham obtido valores de NS superiores a 0,50 considerados satisfatórios por Moriasi et al. (2007). Estudos realizados por Ferreira (2004) indicaram que o TOPMODEL apresenta baixos índices de eficiência para

Tabela 6. Resultados relativos aos eventos de validação do TOPMODEL na Microbacia do Córrego Jaqueira, pertinentes à vazão máxima, lâmina (valores pontuais e erro percentual) e hidrograma de escoamento superficial (coeficiente de eficiência - NS)

\begin{tabular}{|c|c|c|c|c|c|c|c|}
\hline \multirow[b]{2}{*}{ Evento } & \multicolumn{3}{|c|}{ Vazão máxima } & \multicolumn{3}{|c|}{ Lâmina } & \multirow[b]{2}{*}{$\begin{array}{l}\text {-Hidrograma } \\
\text { NS (adim.) }\end{array}$} \\
\hline & $\frac{\text { Simulad }}{1}$ & servada & $\begin{array}{l}\text { Erro } \\
(\%)\end{array}$ & Simulad & $\frac{\text { Observada }}{\mathrm{m} \text { ) }}$ & $\begin{array}{l}\text { Erro } \\
(\%)\end{array}$ & \\
\hline P1 & 1,32 & 6,07 & 78 & 0,09 & 0,23 & 61 & 0,31 \\
\hline P3 & 0,82 & 3,63 & 77 & 0,05 & 0,15 & 67 & 0,03 \\
\hline P5 & 2,21 & 6,30 & 65 & 0,13 & 0,19 & 32 & 0,58 \\
\hline P6 & 2,51 & 2,47 & -2 & 0,14 & 0,09 & -56 & 0,59 \\
\hline P7 & 6,19 & 7,90 & 22 & 0,45 & 0,41 & -10 & 0,79 \\
\hline P8 & 2,87 & 8,78 & 67 & 0,15 & 0,32 & 53 & 0,50 \\
\hline P9 & 1,44 & 5,77 & 75 & 0,14 & 0,41 & 66 & 0,01 \\
\hline P12 & 3,10 & 2,54 & -22 & 0,20 & 0,16 & -25 & 0,73 \\
\hline P14 & 1,18 & 1,00 & -18 & 0,06 & 0,05 & -20 & 0,04 \\
\hline P15 & 0,54 & 1,45 & 63 & 0,03 & 0,05 & 40 & 0,32 \\
\hline P16 & 2,54 & 3,29 & 23 & 0,14 & 0,12 & -17 & 0,81 \\
\hline
\end{tabular}


eventos com precipitação total inferior a $20 \mathrm{~mm}$. Dentre os eventos selecionados para validação os que apresentaram PT $<20 \mathrm{~mm}$ foram P3, P6 e P15 (NS inferior a 0,7). Vale lembrar que o limite de $20 \mathrm{~mm}$ é arbitrário e que Castilho (2005) verificou que o desempenho do TOPMODEL foi melhor para os eventos com PT inferiores a $20 \mathrm{~mm}$. Este é o caso do P6 (NS iguala 0,59 ), com PT igual a $19 \mathrm{~mm}$ e alta intensidade de precipitação, diferentemente de P3 e P6; enfim, tais resultados mostram que se deve evitar a imposição de limites arbitrários com relação às precipitações cujas características resultam em melhores eficiências.
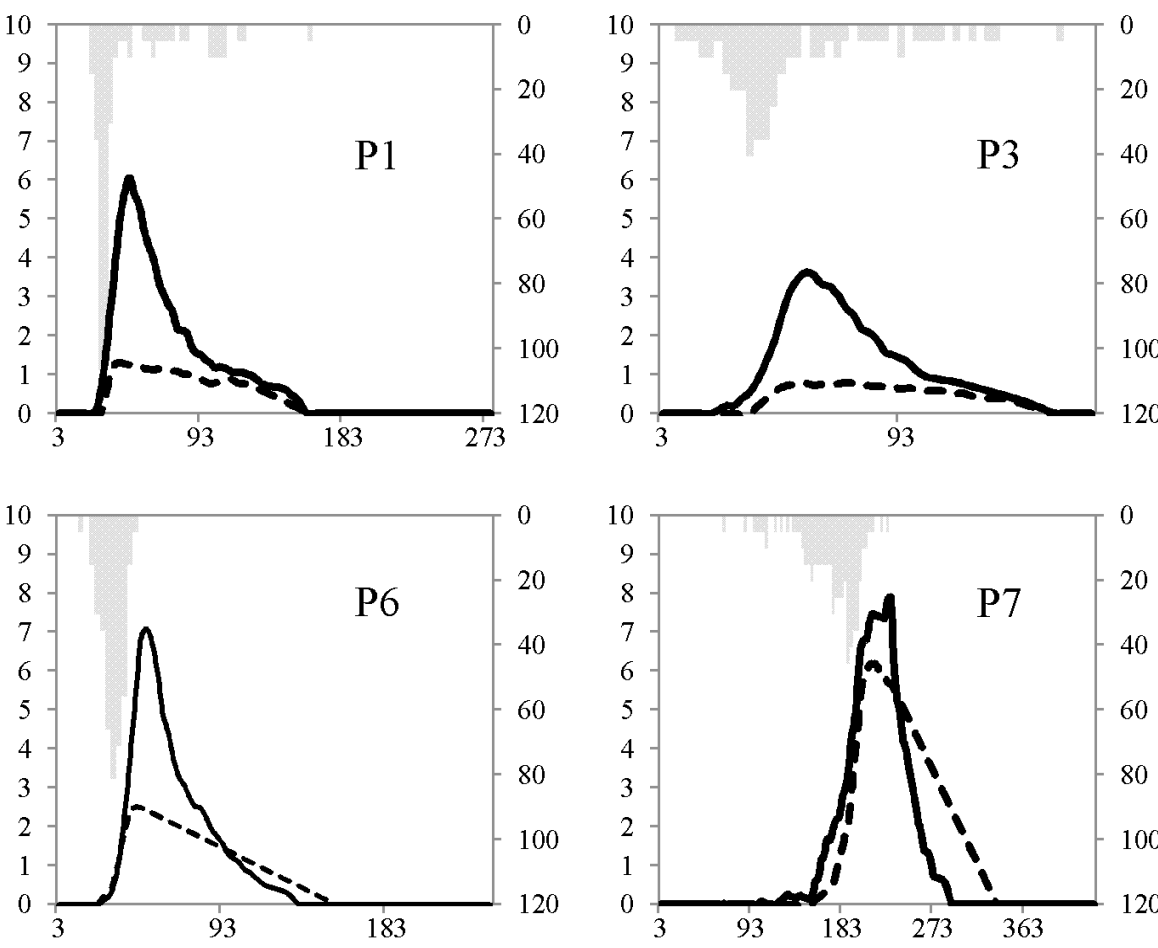

Na Figura 3 são apresentados os hidrogramas observados e simulados referentes aos eventos de precipitação usados na validação do TOPMODEL para a MCJ. Notam-se três grupos de comportamento distintos: Grupo A (P1, P3, P5, P6, P8, P9 e P15.); Grupo B (P12 e P14) e Grupo C (P7 e P16) caracterizados conforme descrito na sequência.

A maior parte dos eventos esteve enquadrada no Grupo A caracterizando uma tendência das simulações para a MCJ. Observou-se que a simulação da hidrógrafa não foi plenamente satisfatória ocorrendo em média uma subestimativa de 50\% em $\mathrm{Q}_{\text {máx }}$ e LES. Observou-se também pequeno deslocamento
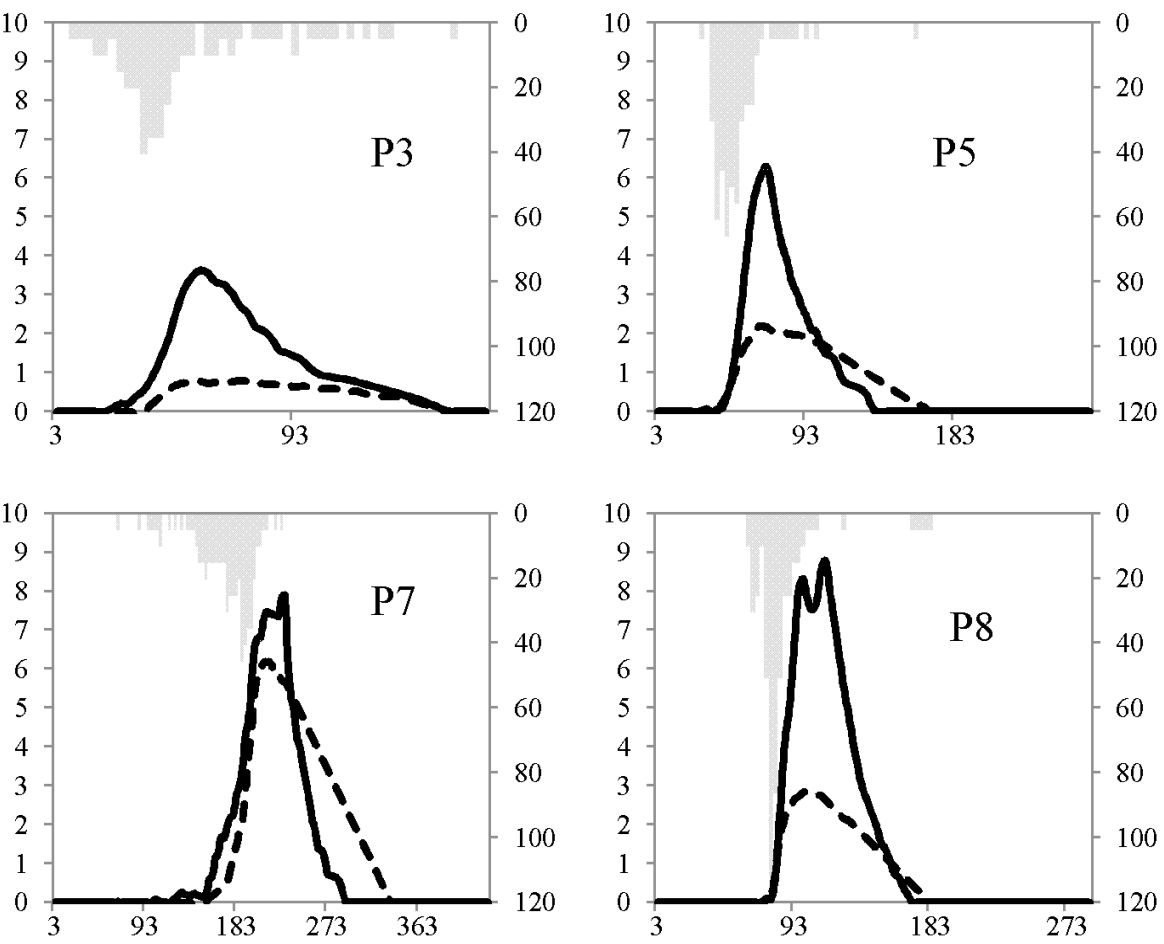
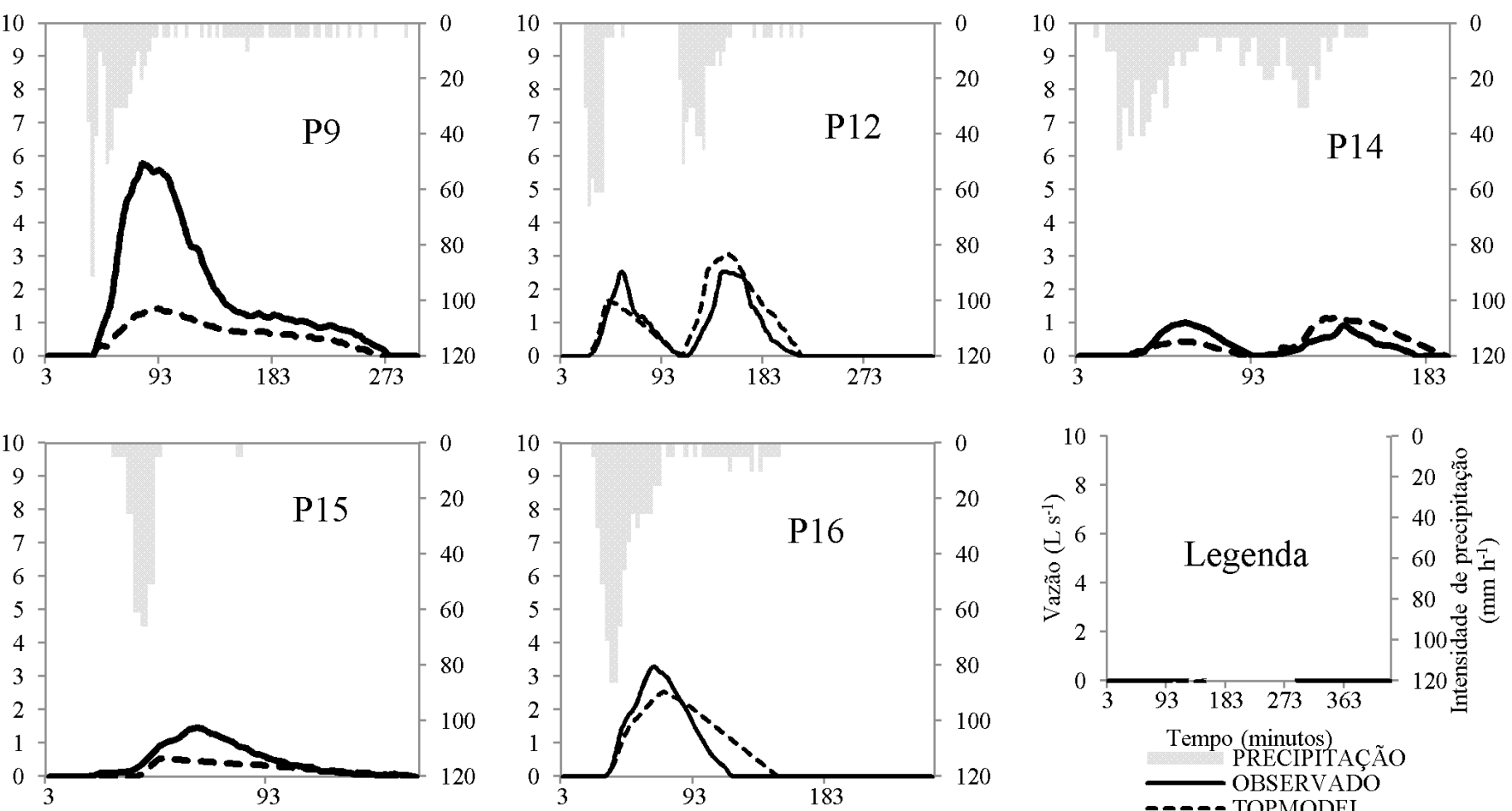

Figura 3. Hidrogramas de escoamento superficial dos eventos usados para validação do TOPMODEL na Bacia Hidrográfica do Córrego Jaqueira 
entre tempo de pico observado e simulado (cerca de $3 \mathrm{~min}$ ), associado à tendência em atrasar o início do escoamento superficial.

Os eventos do Grupo B apresentam simulações com dois picos de vazão máxima havendo subestimativa do primeiro pico (em torno de $43 \%$ ) e superestimativa do segundo (em torno de 26\%). Observa-se, ainda, tendência em atraso no início do escoamento superficial simulado e adiantamento dos picos de vazão máxima simulada.

Os eventos do Grupo C apresentaram os melhores valores de NS com a presença de um único pico de vazão bem definido. Nota-se que os valores observados e simulados estão muito próximos, sendo que os valores de $\mathrm{Q}_{\text {máx }}$ observada e simulada apresentam pequena diferença (em média 21\%).

Apesar de apresentar baixa eficiência $(\mathrm{NS}<0,5)$ em cinco das 11 simulações dos hidrogramas de escoamento superficial, o modelo pode ser considerado promissor na modelagem dos hidrogramas de eventos isolados pois várias são as simplificações envolvidas na aplicação realizada no presente estudo, como a pequena quantidade de eventos disponíveis para calibração e a grande diferença nos usos e propriedades do solo em uma pequena área). Tal afirmação corrobora Nourani et al. (2011), que verificaram ótimo desempenho do modelo em bacia de maiores dimensões. As características conservacionistas do manejo da MCJ não permitem a geração de volumes consideráveis de escoamento superficial, o que justifica, em parte, o desempenho observado no presente estudo.

Outra parte deste desempenho se deve ao TOPMODEL considerar a espacialização apenas do IT e não das características de uso do solo, conforme também relatado por Huiping \& Shufen (2010), sendo a bacia simplificada em um sistema simples em que a única variabilidade espacial modelada é a do IT. As diferentes formas de uso e ocupação do solo na MCJ levam a valores discrepantes das propriedades associadas ao movimento de água no solo, notadamente sobre a infiltração (Tabela 1) influindo diretamente na maior parte dos parâmetros de entrada $\left(\mathrm{m}_{0}, \mathrm{~T}_{0}, \mathrm{SR}_{\text {máx }}, \mathrm{SR}_{\text {init }}\right.$ e ChVel). Tais parâmetros são tomados apenas como uma média ponderada em função da área ocupada por cada uso, o que pode levar o modelo a não representar, de forma correta, o movimento superficial e subterrâneo da água no solo em toda a bacia. Em especial se destaca o $\mathrm{T}_{0}$, que é diretamente dependente da condutividade hidráulica do solo (Beven, 1984; Huiping \& Shufen, 2010), a qual é próxima à taxa de infiltração estável e que varia, na MCJ, de 50,1 a 439,1 $\mathrm{mm} \mathrm{h}^{-1}$ (Tabela 1 ). Acredita-se que o desempenho do TOPMODEL seria melhor em condições mais homogêneas das características edáficas e de uso do solo. Todavia, os resultados apresentados mostram, ainda assim, que o modelo pode ser aplicado em condições de diversos usos em uma mesma bacia.

Finalmente, mais uma parte do desempenho é explicado conforme também relatado por Bhaskar et al. (2005), pelo conjunto de eventos usados na calibração. Aparentemente, os parâmetros de entrada do TOPMODEL (sobretudo $\mathrm{m}_{0}$ e $\mathrm{T}_{0}$ ) dependem bastante dos eventos de chuva usados na calibração. Para um desempenho melhor do TOPMODEL, seria recomendável a realização da calibração para diferentes conjuntos de eventos de calibração, ou seja, calibrações distintas para eventos de chuva com características distintas quanto ao perfil de precipitação, lâmina precipitada e intensidades de precipitação porém, para a realização de tais calibrações seria necessário um conjunto maior de hidrogramas e pluviogramas medidos.

\section{Conclusões}

1. O TOPMODEL mostrou tendência em subestimar $\mathrm{Q}_{\text {máx }}$ e LES na maioria dos eventos na microbacia em manejo conservacionista em estudos.

2. Os valores de NS positivos demonstraram que o TOPMODEL é promissor na modelagem dos hidrogramas de escoamento superficial de eventos de chuva isolados na microbacia com manejo conservacionista em estudos.

\section{Agradecimentos}

Ao Conselho Nacional de Desenvolvimento Científico e Tecnológico (CNPq) pelo apoio financeiro (Processo 484587/2011-0) e Bolsas de Produtividade em Pesquisa e à Fundação de Amparo à Pesquisa do Espírito Santo (FAPES) pelo auxílio Taxa de Pesquisa (Processo 59941456/2012).

\section{Literatura Citada}

Beven, K. Infiltration into a class of vertically non-uniform soils. Hydrological Sciences Journal, v.29, p.425-434, 1984. http:// dx.doi.org/10.1080/02626668409490960

Beven, K.; Freer, J. A dynamic TOPMODEL. Hydrological Processes, v.15, p.1993-2011, 2001. http://dx.doi.org/10.1002/hyp.252

Beven, K. J.; Kirkby, M. J. A physically based, variable contributing area model of basin hydrology. Hydrological Sciences Bulletin, v.24, p.43-69, 1979. http://dx.doi.org/10.1080/02626667909491834

Beven, K. J.; Kirkby, M. J.; Schofield, N.; Tagg, A. F. Testing a physically-based flood forecasting model (TOPMODEL) for three U.K. catchments. Journal of Hydrology, v.69, p.119-143, 1984. http://dx.doi.org/10.1016/0022-1694(84)90159-8

Bhaskar, N. R.; Brummett, L. K.; French, M. N. Runoff modeling of a mountainous catchment using TOPMODEL: A case study. Journal of the American Water Resources Association, v.41, p.107-121, 2005. http://dx.doi.org/10.1111/j.1752-1688.2005.tb03721.x

Brandão, V. dos S.; Cecílio, R. A.; Pruski, F. F.; Silva, D. D. da. Infiltração da água no solo. 3.ed. Viçosa: UFV, 2006. 120p.

Buytaert, W.; Célleri, R.; Biévre, B. de; Deckers, J.; Wyseure, G. Modelando el comportamiento hidrológico de microcuencas de páramo em el Sur Del Ecuador com TOPMODEL. Revista Marginalia, v.6, p.149-157, 2003.

Castilho, C. P. G. Simulação hidrologica de uma bacia rural utilizando o modelo TOPMODEL acoplado a um modelo de interceptação de chuva pela cobertura vegetal. Campinas: UNICAMP, 2005. 280p.

EMBRAPA - Empresa Brasileira de Pesquisa Agropecuaria. Manual de métodos de análises de solo. 2.ed. Rio de Janeiro: CNPS, 1997. $212 \mathrm{p}$.

Ferreira, L. Simulação hidrológica utilizando o modelo TOPMODEL em bacias rurais, estudo de caso na Bacia do Ribeirão dos Marins - Seção Monjolinho (SP). Campinas: UNICAMP, 2004. 205p. 
Franchini, M.; Wendling, J.; Obled, C.; Todini, E. Physical interpretation and sensitivity analysis of the TOPMODEL. Journal of Hydrology, v.175, p.293-338, 1996. http://dx.doi.org/10.1016/ S0022-1694(96)80015-1

Gumindoga, W.; Rwasoka, D. T.; Murwira, A. Simulation of streamflow using TOPMODEL in the Upper Save River catchment of Zimbabwe. Physics and Chemistry of the Earth, Parts A/B/C, v.36, p 806-813, 2011.

Huiping, D.; Shufen, S. Extension of TOPMODEL applications to the heterogeneous land surface. Advances in Atmospheric Sciences, v.27, p.164-176, 2010. http://dx.doi.org/10.1007/s00376-0098146-z

Kulasova, A.; Beven, K. J.; Blazkova, S. D.; Rezacova, D.; Cajthaml, J. Comparison of saturated areas mapping methods in the Jizera Mountains, Czech Republic. Journal of Hydrolougy \& Hydromechanics, v.62, p.160-168, 2014. http://dx.doi. org/10.2478/johh-2014-0002

Lima, J. S. de S.; Silva, S. de A.; Oliveira, R. B. de; Cecílio, R. A.; Xavier, A. C. Variabilidade temporal da precipitação mensal em Alegre, ES. Revista Ciência Agronômica, v.39, p.327-332, 2008.

Lin, K.; Zhang, Q.; Chen, X. An evaluation of impacts of DEM resolution and parameter correlation on TOPMODEL modeling uncertainty. Journal of Hydrology, v.394, p.370-383, 2010. http:// dx.doi.org/10.1016/j.jhydrol.2010.09.012

Mine, M. R. M.; Clarke, R. T. O uso do Topmodel em condições brasileiras: resultado preliminar. Revista Brasileira de Recursos Hídricos, v.1, p.89-105, 1996.

Moriasi, D. N.; Arnold, J. G.; Liew, M. W. van; Bingner, R. L.; Harmel, R. D.; Veith, T. L. Model evaluation guidelines for systematic quantification of accuracy in watershed simulations. Transactions of the ASABE, v.50, p.885-900, 2007. http://dx.doi. org/10.13031/2013.23153

Nash, J. E.; Sutcliffe, J. V. River flow forecasting through conceptual models part I - A discussion of principles. Journal of Hydrology, v.10, p.282-290, 1970. http://dx.doi.org/10.1016/00221694(70)90255-6

Nourani, V.; Roughani, A.; Gebremichael, M. Topmodel capability for rainfall-runoff modeling of the ammameh watershed at different time scales using different terrain algorithms. Journal of Urban and Environmental Engineering, v.5, p.1-14, 2011. http://dx.doi. org/10.4090/juee.2011.v5n1.001014
Pan, F.; King, A. W. Downscaling 1-km topographic index distributions to a finer resolution for the TOPMODEL-Based GCM hydrological modeling. Journal of Hydrologic Engineering, v.17, p.243-251, 2012. http://dx.doi.org/10.1061/(ASCE)HE.1943-5584.0000438

Qi, W.; Zhang, C.; Chu, J.; Zhou, H. Sobol's sensitivity analysis for TOPMODEL hydrological model: A case study for the Biliu River Basin, China. Journal of Hydrology and Environment Research, v.1, p.1-10, 2013.

Quinn, P. F.; Beven, K. J.; Lamb, R. The $\ln (\mathrm{a} / \tan / \beta)$ index: How to calculate it and how to use it within the topmodel framework. Hydrological Processes, v.9, p.161-182, 1995. http://dx.doi. org/10.1002/hyp.3360090204

Santos, I.; Kobiyama, M. Aplicação do TOPMODEL para determinação de áreas saturadas da bacia do rio Pequeno, São José dos Pinhais, PR, Brasil. Ambiente \& Agua - An Interdisciplinary Journal of Applied Science, v.3, p.77-89, 2008.

Schuler, A. E.; Moraes, J. M.; Milde, L. C.; Groppo, J. D.; Martinelli, L. A.; Victoria, R. L.; Calijuri, M. L. Análise da representatividade física dos parâmetros do TOPMODEL em uma bacia de mesoescala localizada nas cabeceiras do Rio Corumbataí, São Paulo. Revista Brasileira de Recursos Hídricos, v.5, p.5-25, 2000.

Siefert, C. A. C. Delimitação de áreas hidrologicamente sensíveis por meio de modelagem de processos e da relação solo-vegetação em ambientes hidromórficos. Curitiba: UFPR, 2012. 111p.

Silva, R. V.; Kobiyama, M. Estudo comparativo de três formulações do TOPMODEL na Bacia do Rio Pequeno, São José dos Pinhais, PR. Revista Brasileira de Recursos Hídricos, v.12, p.93-105, 2007.

Varella, R. F.; Campana, N. A. Simulação matemática do processo de transformação de chuva em vazão: Estudo do modelo TOPMODEL. Revista Brasileira de Recursos Hídricos, v.5, p.121-139, 2000.

Wu, S.; Li, J.; Huang, G. H. Modeling the effects of elevation data resolution on the performance of topography-based watershed runoff simulation. Environmental Modelling \& Software, v.22, p.1250-1260, 2007. http://dx.doi.org/10.1016/j. envsoft.2006.08.001

Zanetti, S. S.; Oliveira, V. de P. S. de; Sousa, E. F.; Almeida, F. T. de; Sugawara, M. T.; Silva, J. M. A. da. Modelagem hidrológica em microbacia hidrográfica parte II: Teste do modelo HidroBacia. Revista Brasileira de Engenharia Agrícola e Ambiental, v.13, p.435442, 2009. http://dx.doi.org/10.1590/S1415-43662009000400010 